3. Colovic, R. \& Perisic-Savic, M. Retroperitoneal biloma secondary to operative common bile duct injury. Hepatobiliary Surg 1991, 3: 193-197.

4. Achong, D.M., Newman, J.S. \& Oates, E. False negative morphine augmented cholescintography: a case of subacute gallbladder perforation. J Nucl Med 1992, 33: 256-257.
5. Dupas, J.-L., Mancheron, H., Senevet, F., Delamarre, J., Delcenserie, R. \& Capron, J.-P. Hepatic subcapsular biloma. An unusual complication of endoscopic cholangiopancreatography. Gastroenterology 1988, 94: 1225-1227.

\title{
Osteomyelitis of symphysis pubis following renal transplantation
}

\author{
R.M. Jindal, B. Idelson, D. Bernard and S.I. Cho \\ Transplant Service and Section of Nephrology, The Boston University Medical Center, D-511, The \\ University Hospital, 88 East Newton Street, Boston, MA 02118, USA
}

Summary: We describe what we believe is the first reported case of osteomyelitis of the symphysis pubis following renal transplantation. Computed tomographic and magnetic resonance imaging scans were useful in establishing the diagnosis.

\section{Introduction}

Infection of the symphysis pubis is a rare complication accounting for less than $1 \%$ of cases of osteomyelitis. The predisposing causes reported are pelvic surgery, trauma and intravenous drug abuse. ${ }^{1-3}$ We report what is to our knowledge the first case of osteomyelitis of the symphysis pubis following renal transplantation. This case highlights some of the well-known features of this condition such as delay in diagnosis, insidious onset and inadequacy of plain X-rays to detect early changes in the symphysis pubis. ${ }^{4}$

\section{Case report}

A 58 year old white woman had a one-haploid living-related renal transplantation to the left iliac fossa by standard surgical techniques. Preoperative evaluation of the donor showed fibromuscular dysplasia of the right renal artery, but not on the left. Therefore, it was decided to use the right donor kidney and during transplantation resect the diseased artery and replace it with a saphenous vein

Correspondence: R.M. Jindal, M.D., F.R.C.S.(Ed.), M.Sc.(Oxf.), Department of Transplantation, The Mount Sinai School of Medicine, 1, Gustave Levy Place, New York, NY 10029-6579, USA.

Accepted: 22 February 1993 bypass graft from the left groin. The donor uretert was hooked up to the recipient bladder by Politano-Leadbetter technique. There were no intra-operative complications. Sequential immunosuppression was used, anti-lymphocyte globulin (ALG), azathioprine and prednisone for induction. Cyclosporin was substituted for ALG when the serum creatinine was $<5 \mathrm{mg} / \mathrm{dl}$.

Sonogram and renal scan of the transplanted kidney at days 1 and 5 were normal. A low volume cystogram at day 5 did not show any leak allowing the removal of the Foley catheter. She developed cellulitis in the groin wound at the site of saphenous vein surgery 2 weeks later. Open drainage of the abscess yielded coagulase-positive staphylococci and appropriate antibiotics were given. This was followed by prolonged wound discharge.

She then remained well for 5 months when she presented with a low-grade temperature and a $2 \mathrm{~cm}$ wound abscess at the transplant incision, which o was drained under local anaesthesia. The wound $N$ continued to discharge for 3 weeks despite ade- N quate antibiotics and local wound dressing. At this stage she complained of suprapubic discomfort for which a plain X-ray of the pelvis, ultrasound of the transplanted kidney and pelvis and urine culture were obtained, but did not reveal any abnormality.

Approximately 6 weeks later, she presented with a high grade fever, severe pain over the symphysis 
pubis, adductor spasm and a waddling gait. Computed tomography (CT) (Figure 1) and magnetic resonance imagery (MRI) (Figure 2) of the pelvis showed multiple sequestrae and erosion of the symphysis pubis. Surgical exploration of the wound under general anaesthesia revealed a superficial wound abscess with a track leading to the pubic symphysis. The track was opened, pus drained and sequestrectomy carried out. Coagulase-positive Staphylococcus aureus was cultured from the pus, blood and sequestra. She was treated with intravenous vancomycin and ceftazidime for 2 weeks, later changed to intravenous nafcillin for a further 6 weeks. This was followed by oral oxycillin for 4 weeks. The wound is now completely closed and she is symptom-free.

\section{Discussion}

Osteomyelitis of the symphysis pubis is an uncommon complication but is being reported with increasing frequency in intravenous drug abusers

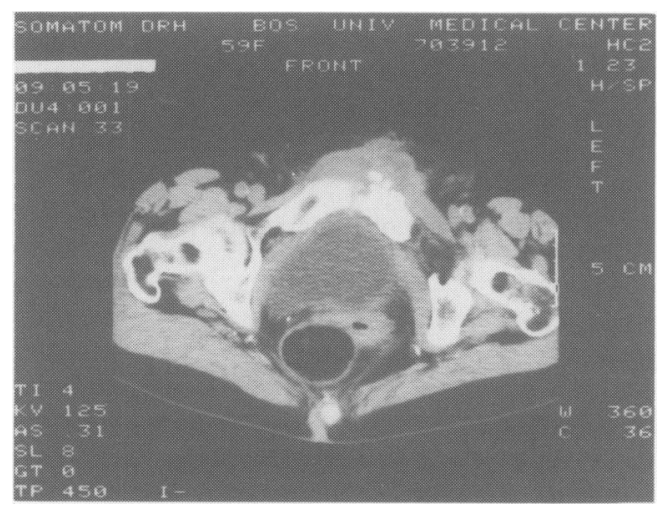

Figure 1 CT scan of the pubic symphysis; there are sequestra in the soft tissue on the left side.

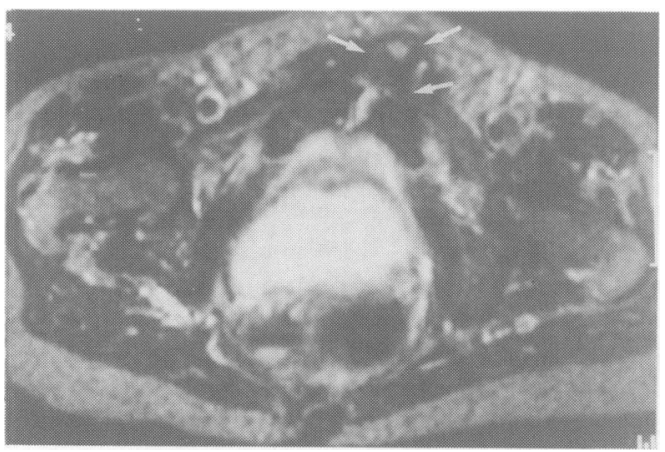

Figure 2 MRI of the pubic symphysis showing soft tissue swelling and bony destruction of the left pubic symphysis (arrowed). and with unusual organisms, either Pseudomonas aeruginosa or Klebsiella aerobacter; about 50 cases have been reported. ${ }^{1,2,5,6}$ It is not certain whether bone or joint involvement is the primary lesion in osteomyelitis of the symphysis pubis. Osteitis pubis may have identical signs and symptoms and initial radiological features may be the same. ${ }^{6}$

Osteomyelitis of the symphysis pubis may be considered in the differential diagnosis of persistent wound infection, pyrexia of unknown origin and urinary infection after renal transplantation. The classical features of pain, tenderness over the pubis, spasms of the adductor muscles and rectus abdominis with a waddling gait may not always be present. Plain X-ray showing irregularity and widening of the symphysis pubis may represent normal variations, and are also seen postpartum and in instability of the symphysis pubis. ${ }^{7}$

Although the occurrence of osteomyelitis of the symphysis pubis after external trauma is obvious, the mechanism of osteomyelitis after pelvic and urological procedures is still not clear. Steinbach $e t$ $a l^{8}$ showed the presence of venous obstruction from the pubic bone to the pelvic veins by intraosseous phlebography on postoperative patients after open and retropubic prostatectomy. This obstruction was thought to be due to thrombophlebitis leading to demineralization of the symphysis pubis. Rosenthal et al. ${ }^{9}$ have suggested that during the vesico-urethral suspension operation, a bladder or urethral perforation by a suture could allow a direct communication between the urinary stream and the cartilagenous symphysis. This would lead to osteomyelitis of the symphysis pubis if the urine was infected for any reason.

The development of osteomyelitis pubis in our case was probably due to direct extension of the superficial wound infection to the pubis. It is also possible that a minor leak from the ureteroneocystostomy led to the formation of urinoma which subsequently became infected and spread to the retro-pubic area.

Superficial wound infection spreading by the blood stream and localizing to the symphysis pubis is also a possibility. Although haematogenous osteomyelitis usually occurs at the metaphysis in long tubular bones, it also occurs at the metaphyseal-equivalent locations of which the symphysis pubis is one. ${ }^{10}$ It is most likely that immunosuppression contributed to the development of osteomyelitis in our case, although in over 350 renal transplants performed in our unit, this was the first such case.

Osteomyelitis of the pubis symphysis is perhaps more difficult to detect at an early stage because signs and symptoms are vague and a high degree of clinical suspicion is required. Radiological investigations with plain X-rays, CT, MRI and bone scan are helpful and may be diagnostic. ${ }^{1,7}$ Care 
must be taken with the latter technique to avoid radioisotope accumulation in the bladder urine that can obscure visualization of the pubic bone.

CT and MRI scans have been used for the differential diagnosis of a variety of lesions of the pubis symphysis such as fractures, osteolysis, osteomyelitis and dislocations. Vogelzang et al. ${ }^{11}$ described a case of tuberculous osteomyelitis of the pubis in which CT scan showed irregular destruc-

\section{References}

1. Del Busto, R., Quinn, E.L., Fisher, E.J. \& Madhaven, T. Osteomyelitis of the pubis symphysis. JAMA 1982, 248: 1498-1500.

2. Sequeira, W., Jones, E., Seigel, M., Lorenz, M. \& Kallick, C. Pyogenic infections of the pubis symphysis. Ann Intern Med 1982, 96: 604-606.

3. Burns, J.R. \& Gregory, J.G. Osteomyelitis of the pubis symphysis after urological surgery. J Urol 1977, 118: 800-805.

4. Evans, T.W., Evely, R.S. \& Morcos, S.K. Asymptomatic osteomyelitis of the symphysis pubis. Postgrad Med J 1985, 61: $267-268$.

5. Kido, D., Bryan, D. \& Halpern, M. Haematogenous osteomyelitis in drug addicts. Am J Roentgenol 1973, 118: 356-363.

6. Rutherford, A. Osteomyelitis of the symphysis pubis. $N Z$ Med J 1983, 96: 322-333.

7. Keats, T.E. An Atlas of Normal Roentgen Variants that may Simulate Disease, 2nd ed. Yearbook Medical Publishers, Chicago, 1979, p. 196. tion and erosion of the pubic bone with a soft tissue mass. Plain X-ray had earlier failed to demonstrate the lesion. Manzaneque et al. ${ }^{12}$ also described the use of CT scan to diagnose tuberculous osteomyelitis of the pubic symphysis. Turner et al. ${ }^{13}$ found MRI to be particularly useful in the differential diagnosis of pain in the pubic symphysis in a patient with chloroma.

8. Steinbach, H.L., Patrakis, N.L., Gilfillan, R.S. \& Smith, D.R. The pathogenesis of osteitis pubis. J Urol 1955, 74: 840-846.

9. Rosenthal, R.E., Spickard, W.A., Markham, R.D. \& Rhamy, R.K. Osteomyelitis of the symphysis pubis. J Bone Joint Surg 1982, 64-A: 123-128.

10. Nixon, G.W. Haematogenous osteomyelitis of metaphysealequivalent locations. Am J Roentgenol 1978, 130: 123-129.

11. Vogelzang, R.L., Hendrix, R.W. \& Neiman, H.L. Computed tomography of tuberculous osteomyelitis of the pubis. $J$ Comput Assist Tomogr 1983, 7: 914-915.

12. Manzaneque, L., Martin, I., Garcia-Bragado, F., Beiztegui, A., Dastis, C. \& Sanchez-Matas, P. Osteoarticular tuberculosis of the symphysis pubis presenting as a hypogastric cystic mass in a woman with primary Sjogrens syndrome. $\mathrm{Br} J$ Rheumatol 1992, 31: 495-496.

13. Turner, R.M., Wallace, W.P. \& Prietto, C. MR of soft tissue chloroma in a patient presenting with left pubic pain and hip pain. J Comput Assist Tomogr 1991, 15: 700-702.

\title{
Spontaneous rupture of the spleen in association with idiopathic thrombocytopaenic purpura
}

\author{
M.C. Winslet, M.J. Webberley, V. Melikian ${ }^{1}$ and I.A. Donovan
}

Department of Surgery and ${ }^{1}$ Medicine, Dudley Road Hospital, Dudley Road, Birmingham B18 7QH, UK

Summary: Spontaneous rupture of the spleen is rare and has not been previously reported in association with idiopathic thrombocytopaenic purpura. Its rarity makes familiarity with its presentation difficult and may result in a potentially fatal delay in diagnosis and treatment.

\section{Introduction}

Rupture of a histologically normal spleen usually occurs as a result of indirect trauma or iatrogenic injury. Rarely, in association with well-documented pathological changes, the spleen may

Correspondence: M.C. Winslet, M.S., F.R.C.S., University Department of Surgery, Royal Free Hospital, Pond Street, London NW3 2QG, UK.

Accepted: 4 February 1993 spontaneously rupture. Spontaneous rupture of a histologically normal spleen is exceedingly rare and its association with idiopathic thrombocytopaenic purpura has not been previously reported.

\section{Case report}

A 24 year old male with congenital aortic valve disease and idiopathic thrombocytopaenic purpura 